\title{
Comparative Analysis of Geometrical Properties of Sampling Schemes on the Sphere
}

\author{
Usama Elahi, Zubair Khalid, Member, IEEE, and Rodney A. Kennedy, Fellow, IEEE \\ Research School of Engineering, \\ College of Engineering and Computer Science, \\ The Australian National University, \\ Canberra, ACT 2601, Australia \\ Email: \{usama.elahi,zubair.khalid,rodney.kennedy\}@anu.edu.au
}

\begin{abstract}
In this work, we carry out the comparative analysis of the geometrical properties of the sampling schemes on the sphere. Among the sampling schemes devised on the sphere, we focus on equiangular sampling, Gauss-Legendre (GL) quadrature based sampling, optimal-dimensionality sampling, sampling points of extremal systems and spherical design as these schemes support the accurate representation of the band-limited signals. We analyse sampling efficiency, minimum geodesic distance, mesh norm, mesh ratio and Riesz $s$-energy for these sampling schemes. Since these sampling schemes require different number of samples for the accurate representation of a band-limited signal and therefore have different sampling efficiency, we formulate these geometrical properties to take into account the sampling efficiency for a meaningful comparison. We illustrate that the optimal dimensionality, extremal system and spherical design sampling schemes exhibit desirable geometrical properties. Among these schemes, extremal system sampling scheme has superior geometrical properties. However, the accuracy of the representation of a band-limited signal degrades with the increase in band-limit for extremal system sampling scheme, due to which we propose to use extremal point sampling scheme for small band-limits. We also propose to use optimal dimensional sampling scheme for moderate to large band-limits as it exhibits desirable geometrical properties and has the capability to accurately represent the band-limited signal.
\end{abstract}

Index Terms-Sampling, spherical harmonics, spherical harmonic transform, band-limited signals, 2-sphere (unit sphere).

\section{INTRODUCTION}

Design of sampling schemes on the sphere finds applications in a variety of fields of science and engineering, which include, but not limited to, geodesy [1], computer graphics [2], cosmology [3], astrophysics [4], medical imaging [5], acoustics [6], and wireless communication [7]. For signals on the sphere, harmonic domain is enabled by the well known spherical harmonic transform (SHT) [8]. The ability to accurately and efficiently compute SHT of the signal from its samples taken over sphere is of significant importance for harmonic analysis and signal representation (or reconstruction) in these applications. It is of fundamental importance that the sampling scheme requires less number of samples for the accurate

This work was supported under the Australian Research Council's Discovery Projects funding scheme (project no. DP150101011). computation of SHT [9]. Furthermore, it is also desirable that the samples on the sphere are uniformly distributed and/or well separated on the sphere [10]-[12].

In the literature, many sampling schemes on the sphere have been devised that permit accurate computation of SHT of a band-limited signal [9]-[11], [11], [13]-[18]. Among these sampling schemes, equiangular sampling schemes [13], [15], [16] and Gauss-Legendre (GL) quadrature based sampling allow theoretically exact computation of SHT. However, these schemes exhibit poor geometrical properties, as we show later in the paper, primarily, due to the dense sampling near the poles. There are other sampling schemes which rely on the quadrature rules on the sphere in order to compute SHT [10], [11]. These schemes have well separated points and therefore the sampling points exhibit superior geometrical properties. However, the computation of SHT for these schemes is computationally intensive and the accuracy of SHT of the band-limited signal degrades with increase in the band-limit of the signal. Geometric properties such as sampling efficiency, minimum geodesic distance, mesh norm, mesh ratio and Riesz $s$-energy of the set of sampling points give an insight of the nature of distribution of points on the sphere [10]-[12], [19], [20]. These geometrical properties also enable us to analyse uniform distribution, dense sampling, regularity and flexibility in the samples placement for a given set of sampling points. The geometrical properties of different sampling schemes on the sphere have been analysed [10], [11] and bounds have been derived on geometrical properties [12], [19], [20]. To the best of our knowledge, the comparative analysis of the geometrical properties of sampling schemes has not been carried out in the literature.

In this paper, we compare the geometrical properties: sampling efficiency, mesh norm, mesh ratio, minimum geodesic distance and Riesz $s$-energy for the sampling schemes: efficient equiangular sampling [15], optimal dimensionality sampling [9], GL quadrature based sampling [21], sampling points of extremal systems [10] and spherical designs [11]. Since the sampling schemes under consideration require different number of samples for accurate representation of a bandlimited signal, we incorporate the sampling efficiency, defined as the degrees of freedom in harmonic space to represent a band-limited signal to the number of samples required on 
the sphere to accurately represent a band-limited signal, into the formulation of the geometrical properties for consistent comparison of the properties of different sampling schemes. Our analysis show that extremal system of points exhibits superior geometrical properties. We also show that spherical designs and optimal dimensionality schemes also have desirable geometrical properties. Since the geometric properties depend on the distribution of points, their analysis give us an insight into the structure of the sampling schemes being considered in the paper.

We structure the remainder of the paper as follows. The necessary mathematical background and notation adopted in this work are presented in Section II. In Section III, we briefly review the sampling schemes under consideration. In section IV, we formulate the geometrical properties and carry out the comparative analysis. Finally, concluding remarks are made in Section V.

\section{MAThematicAl PRELIMinaries}

In this section, we present the necessary mathematical background for signals defined on the sphere.

\section{A. Signals on the Sphere}

We consider square integrable complex functions of the form $g(\hat{\boldsymbol{x}})$ defined on the unit sphere, denoted by $\mathbb{S}^{2} \triangleq\left\{\hat{\boldsymbol{x}} \in \mathbb{R}^{3}:|\hat{\boldsymbol{x}}|=1\right\}$. Here $\hat{\boldsymbol{x}}=\hat{\boldsymbol{x}}(\theta, \phi)=$ $(\sin \theta \cos \phi, \cos \theta \cos \phi, \cos \theta) \in \mathbb{S}^{2} \subset \mathbb{R}^{3}$ denotes a point on the sphere, where $\theta \in[0, \pi]$ denotes the co-latitude and $\phi \in[0,2 \pi)$ is the longitude. The inner product of the two functions $g(\theta, \phi)$ and $h(\theta, \phi)$ defined on $\mathbb{S}^{2}$ is given by [8]

$$
\langle g, h\rangle \triangleq \int_{\mathbb{S}^{2}} g(\theta, \phi) \overline{h(\theta, \phi)} \sin \theta d \theta d \phi,
$$

where $\overline{(\cdot)}$ represents the complex conjugate operation and $\sin \theta d \theta d \phi$ is the differential area element on the sphere. The inner product given in (1) induces a norm $\|g\| \triangleq\langle g, g\rangle^{1 / 2}$. We refer to the functions with finite induced norms as signals on the sphere $\mathbb{S}^{2}[8]$.

\section{B. Spherical Harmonics}

Spherical harmonics [8], $Y_{\ell}^{m}(\theta, \phi)$ of all integer degrees $\ell \geq 0$ and integer orders $m \leq|\ell|$ form a complete orthonormal set of basis functions, and therefore we can expand as

$$
g(\theta, \phi)=\sum_{\ell=0}^{\infty} \sum_{m=-\ell}^{\ell}(g)_{\ell}^{m} Y_{\ell}^{m}(\theta, \phi),
$$

where $(g)_{\ell}^{m}$ is the spherical harmonic coefficient of degree $\ell$ and order $m$ and is given by the spherical harmonic transform (SHT) defined as

$$
(g)_{\ell}^{m} \triangleq\left\langle g, Y_{\ell}^{m}\right\rangle=\int_{\mathbb{S}^{2}} g(\theta, \phi) \overline{Y_{\ell}^{m}(\theta, \phi)} \sin \theta d \theta d \phi,
$$

The signal $g(\theta, \phi)$ is considered as band-limited at degree $L$ if $(g)_{\ell}^{m}=0$ for all $\ell \geq L$. For the representation of a band-limited signal using (2), the summation over degree $\ell$ is truncated at $L-1$. We note that the set of bandlimited signals forms an $L^{2}$ dimensional subspace of $L^{2}\left(\mathbb{S}^{2}\right)$, which we denote by $\mathcal{H}_{L}$.

\section{SAMPling SCHEMES ON THE SPHERE}

In this work, we focus on the recently developed sampling schemes on the sphere which permit accurate computation of SHT of a bandlimited signal from its samples. We first review these sampling schemes before analysing their geometrical properties in the next section. For a signal band-limited at $L$, we use $N$ to denote the spatial dimensionality, that is the number of samples, required by each of the sampling scheme to compute SHT or equivalently represent the band-limited signal accurately.

\section{A. Guass-Legendre Quadrature based Sampling}

This sampling scheme is devised on the basis of the well known Gauss-Legendre quadrature on the sphere [21] and is therefore referred to as Gauss-Legendre (GL) sampling scheme. The GL quadrature is used to construct a sampling theorem such that the SHT of a band-limited signal can be exactly computed from its samples. For a signal band-limited at $L$, this scheme takes samples on $L$ iso-latitude rings with $2 L-1$ equiangular placed samples along longitude $\phi$, resulting in a total requirement of $N_{\mathrm{GL}}=L(2 L-1)$ samples, for the exact computation of SHT. The location of the rings along colatitude $\theta$ is given by the roots of the Legendre polynomials of order $L$ as dictated by the Gauss-Legendre quadrature to discretize the integral given in (3). The variants of the GaussLegendre quadrature scheme have also been proposed (e.g., [22]) that require less number of samples. However, these sampling schemes do not support exact or sufficiently accurate computation of SHT. As an example, the samples on the sphere for GL sampling scheme are shown in Fig.1(a) for $L=10$.

\section{B. Equiangular Sampling}

For the exact computation of SHT of a signal band-limited at $L$, an equiangular scheme was first proposed in [13] which requires $2 L$ iso-latitude equiangular spaced rings of samples with $2 L$ equiangular samples along longitude $\phi$. Recently, an equiangular scheme has been developed [15] that takes samples on an equiangular grid defined by the following sample positions:

$$
\theta_{t}=\frac{\pi(2 t+1)}{(2 L-1)}, \quad t=0,1,2, \ldots, L-1,
$$

and

$$
\phi_{k}=\frac{2 \pi k)}{(2 L-1)}, \quad k=0,1,2, \ldots, 2 L-2,
$$

that is, it requires $L-1$ iso-latitude rings with $2 L-1$ samples along longitude in each ring and a sample at one of the poles $(\theta=0$ or $\theta=\pi)$ and therefore reduces the number of equiangular samples required for the computation of SHT by a factor of two. In total, the number of samples required by equiangular scheme are $N_{\mathrm{E}}=(L-1)(2 L-1)+1$, that is, $3(L-1)$ fewer samples in comparison to the GL scheme. As an example, the samples on the sphere for equiangular sampling scheme are shown in Fig.1(b) for $L=10$. 


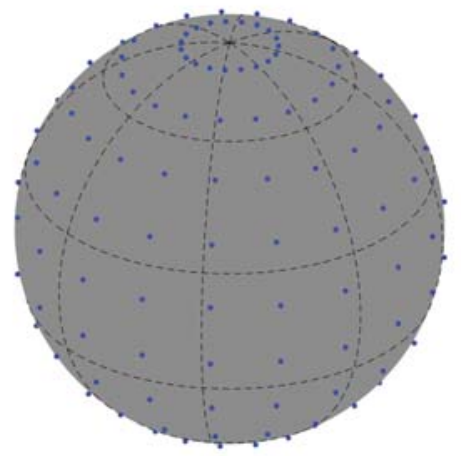

(a) Gauss-Legendre sampling

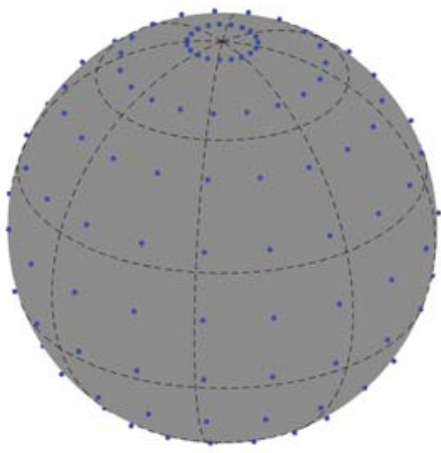

(b) Equiangular sampling

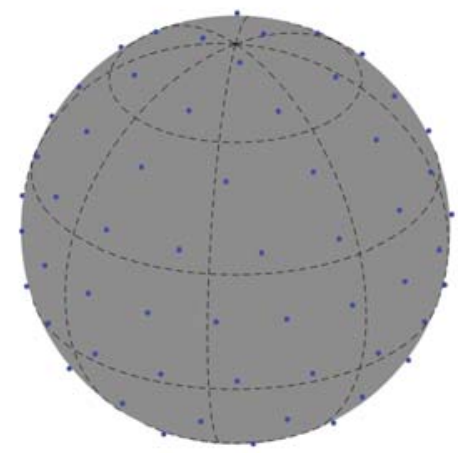

(c) Optimal-dimensionality sampling

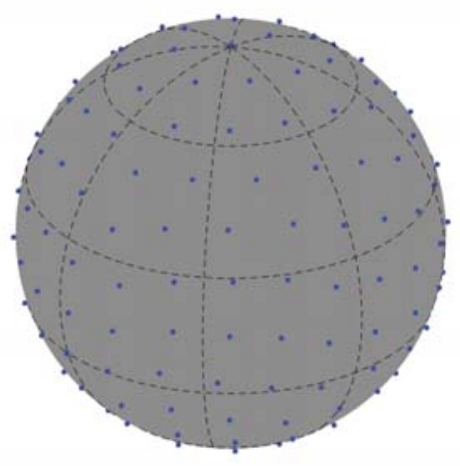

(d) Spherical design sampling

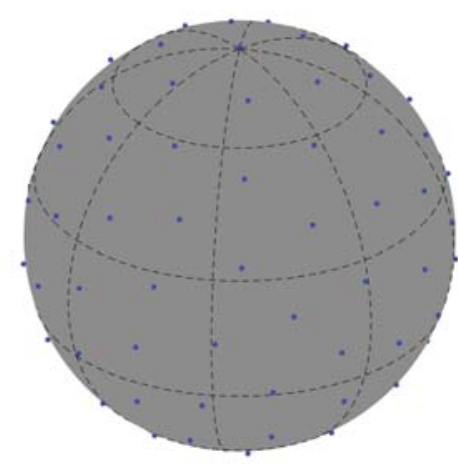

(e) Samples of extremal points

Fig. 1: The sampling schemes on the sphere, (a) Guass-Legendre quadrature based sampling, (b) equiangular sampling, (c) optimal dimensionality sampling scheme, (d) spherical desings and (e) extremal points, presented in Section III for the representation of the signal band-limited at $L=10$.

\section{Optimal-Dimensionality Sampling Scheme}

The spatial dimensionality of both GL and equiangular sampling schemes is twice the optimal spatial dimensionality given by the dimensionality of $\mathcal{H}_{L}$, that is, the degree of freedom in harmonic space to represent a signal bandlimited at spherical harmonic degree $L$. Recently, an optimaldimensionality sampling scheme [9] has been proposed that requires $L^{2}$ points to compute the accurate SHT for signals band-limited at $L$ and therefore the scheme has optimal spatial dimensionality. Although the SHT associated with the optimal dimensionality sampling is not theoretically exact, the accuracy of the SHT has been demonstrated for band-limits up to $L=2048$ with errors on the order of numerical precision. Like GL and equiangular sampling schemes, it is also an isolatitude sampling scheme of the sphere and takes $L$ rings along each latitude. Let $\theta_{k}, k=0,1, \cdots, L-1$ denotes the sample position of the ring along latitude, where these sample locations are chosen such that the accuracy of the computation of SHT is maximized. For a ring placed at $\theta_{k}$, the samples in the ring along $\phi$ are given by

$$
\phi_{n}^{k}=\frac{2 \pi n}{2 k+1}, \quad n=0,1, \ldots, 2 k
$$

that is $2 k+1$ equiangular samples. In total, the number of samples required by optimal-dimensionality sampling scheme is

$$
N_{\mathrm{O}}=\sum_{k=0}^{L-1}(2 k+1)=L^{2}
$$

As an example, the samples on the sphere for optimal dimensionality sampling scheme are shown in Fig.1(c) for $L=10$.

\section{Spherical Designs}

A set of points on the sphere is called a spherical design such that the integral of the signal of maximum spherical polynomial degree $t$ or maximum band-limit $t+1$ over the sphere can be evaluated as an average value over the samples of the signal [11]. Since the spherical design is parameterized by $t$, the set of points is often referred to as spherical $t$-design. Spherical $t$-designs, by definition, enable exact evaluation of the integral of polynomial of maximum degree $t$. For the 
computation of SHT using the points given by spherical design, we first note that the SHT requires to evaluate the integral given in (3), where the integrand is the product of a signal band-limited at $L$ and spherical harmonic $Y_{\ell}^{m}(\theta, \phi)$. Since we require to evaluate the integral for all $\ell<L$, $|m| \leq \ell$, the maximum polynomial degree of integrand is $2 L-2$. Consequently, we require $(2 L-2)$-spherical design for the sampling of band-limited signal such that the SHT can be computed accurately. In our work, we choose the spherical $t$-designs ${ }^{1}$ [11] which takes $t^{2} / 2+t+O(1)$ samples. We use $N_{\mathrm{SD}}$ to denote the number of samples of $(2 L-2)$-spherical design. Due to high computational cost associated with the computation of spherical design, we note that the spherical $t$-designs have been proposed for maximum $t=180$ and therefore the SHT can be computed for band-limits up to $L=91$. As an example, the samples of the $(2 L-2)$-spherical design are shown in Fig.1(d) for $L=10$.

\section{E. Extremal Points on the Sphere}

For a given band-limit $L$, the extremal (maximum determinant) systems are sets of $L^{2}$ extremal points on the sphere which, by definition, maximize the determinant of a basis matrix (see [10] for details). For spherical harmonic basis, extremal points are supported by interpolatory cubature rule with positive weights and therefore enables the accurate computation of SHT of a signal band-limited at $L$ using $N_{\mathrm{ES}}=L^{2}$ sampling points of extremal system. We analyse the accuracy of SHT computation later in the paper. The sampling scheme based on the points ${ }^{2}$ of the extremal system will be referred to as extremal system sampling scheme. As an example, the extremal points on the sphere are shown in Fig.1(e) for $L=10$.

\section{Analysis of Geometrical Properties}

For each of the sampling scheme on the sphere presented in previous section, we here analyse the geometrical properties of the sampling scheme and review the accuracy and computational complexity of the SHT associated with each of the sampling scheme.

\section{A. Sampling Efficiency}

The sampling efficiency, defined as a ratio of the dimensionality of the subspace formed by the band-limited signals, that is the number of coefficients required to represent a band-limited signal in the harmonic domain, to the number of samples required to accurately compute SHT, is the fundamental property of any sampling scheme. For a band-limit $L$, we define the sampling efficiency, denoted by $E_{L}$, of any sampling scheme as a ratio of the dimension of the subspace $\mathcal{H}_{L}$ formed by the band-limited signals to the number of samples, denoted by $N$, required to compute SHT of a band-limited signal $f \in \mathcal{H}_{L}$. It is evident that the optimal dimensionality sampling and

\footnotetext{
${ }^{1}$ The spherical designs are available at http://web.maths.unsw.edu.au/ $\mathrm{rsw} /$ Sphere/EffSphDes/index.html.

${ }^{2}$ We use the the points of extremal systems publicly available at http://web. maths.unsw.edu.au/ $\mathrm{rsw} /$ Sphere/Extremal/New/extremal1.html.
}

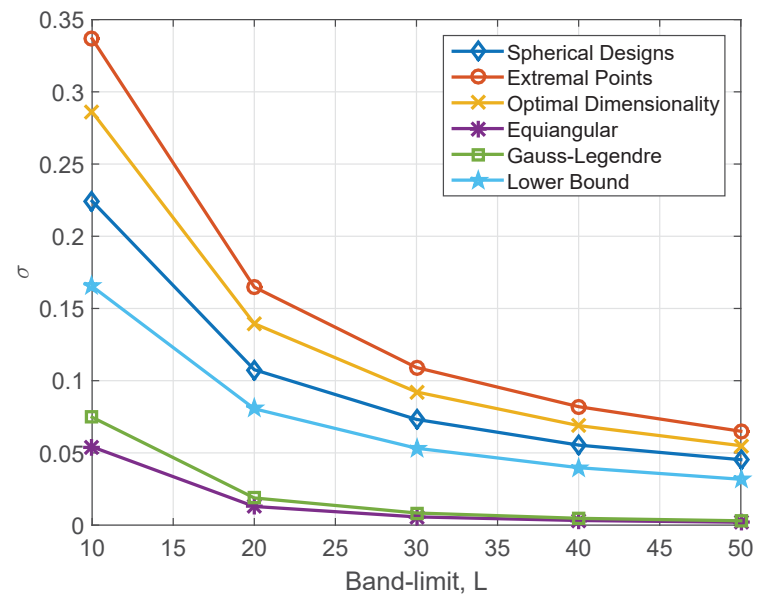

Fig. 2: Normalized minimum geodesic distance $\sigma_{n}(\mathfrak{S})$, given in (10) for band-limits $10 \leq L \leq 50$.

extremal points attain almost the twice (exactly as $L \rightarrow \infty$ ) of the sampling efficiency achieved by equiangular, GL and spherical designs sampling schemes.

\section{B. Minimum Geodesic Distance and Packing Radius}

For a set of sampling points on the sphere, the minimum geodesic distance is defined as the minimum distance between any two points in the set. It is also defined as twice the packing radius on the sphere. It is desirable to design a sampling scheme on the sphere such that the minimum geodesic distance is maximized (well-known sphere packing problem). For points of an extremal system for a band-limit $L$, the minimum geodesic distance is lower bounded by $\pi / 2(L-1)$ [19]. For a set of sampling points on the sphere denoted by $\mathfrak{S}$, the minimum geodesic distance is defined as

$$
\sigma(\mathfrak{S}) \triangleq \min _{\hat{\boldsymbol{x}}, \hat{\boldsymbol{y}} \in \mathfrak{S}} \Delta(\hat{\boldsymbol{x}}, \hat{\boldsymbol{y}}),
$$

where $\Delta(\hat{\boldsymbol{x}}, \hat{\boldsymbol{y}})$ denotes the geodesic (great circle or spherical) distance between two points $\hat{\boldsymbol{x}}\left(\theta_{x}, \phi_{x}\right)$ and $\hat{\boldsymbol{y}}\left(\theta_{y}, \phi_{y}\right)$ and is given by

$$
\begin{aligned}
\Delta(\hat{\boldsymbol{x}}, \hat{\boldsymbol{y}}) & =\cos ^{-1}(\hat{\boldsymbol{x}} \cdot \hat{\boldsymbol{y}}) \\
& =\cos ^{-1}\left(\cos \theta_{x} \cos \theta_{y}+\sin \theta_{x} \sin \theta_{y} \cos \left(\phi_{x}-\phi_{y}\right)\right) .
\end{aligned}
$$

Since the sampling schemes under consideration do not have the same sampling efficiency, we need to incorporate sampling efficiency in defining the minimum geodesic distance for a meaningful comparison of different sampling schemes. We define the normalized minimum geodesic distance as

$$
\sigma_{n}(\mathfrak{S}) \triangleq \frac{1}{E_{L}} \sigma(\mathfrak{S})=\frac{1}{E_{L}} \min _{\hat{\boldsymbol{x}}, \hat{\boldsymbol{y}} \in \mathfrak{S}} \Delta(\hat{\boldsymbol{x}}, \hat{\boldsymbol{y}}) .
$$

Analysis: For each of the sampling schemes presented in Section III, we plot the normalized minimum geodesic $\sigma_{n}(\mathfrak{S})$ for different band-limits $10 \leq L \leq 50$ in Fig. 2, where it can be observed that extremal system of points, spherical design 


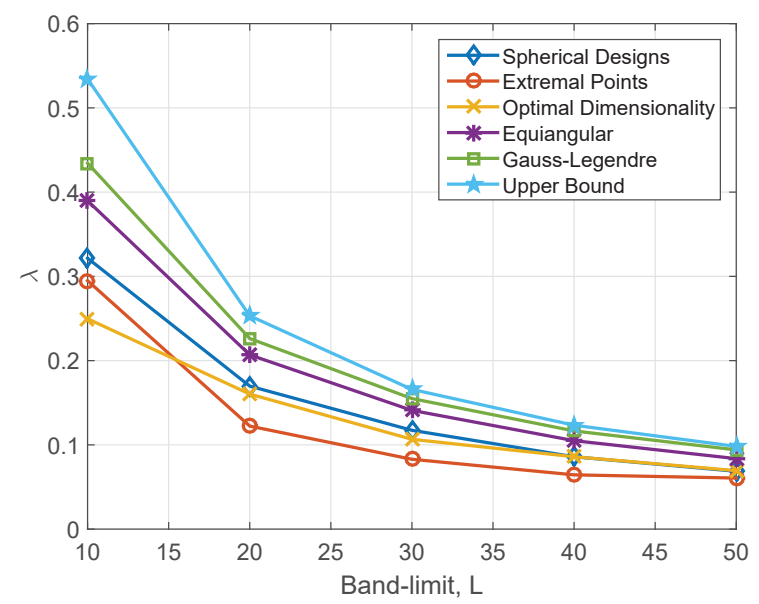

Fig. 3: Mesh norm $\lambda(\mathfrak{S})$, given in (11), for band-limits $10 \leq$ $L \leq 50$ and different sampling schemes.

and optimal dimensionality, all have well separated points on the sphere. The nomralized minimum geodesic distance curves, obtained by using the points of equiangular and GaussLegendre quadrature based sampling schemes, are well below the lower bound values for all degrees $10 \leq L \leq 50$. It is because, these sampling schemes exhibit dense sampling near the poles.

\section{Mesh Norm}

For a set $\mathfrak{S}$ of points on the sphere, mesh norm is defined as the largest geodesic distance from a point $\hat{\boldsymbol{x}} \in \mathbb{S}^{2}$ to the nearest point in the set $\mathfrak{S}$. For a set $\mathfrak{S}$ of sampling points of the sampling scheme parameterized by $L$, we define the mesh norm as

$$
\lambda(\mathfrak{S}) \triangleq \frac{1}{E_{L}} \max _{\hat{\boldsymbol{x}} \in \mathbb{S}^{2}} \min _{\hat{\boldsymbol{y}} \in X} \Delta(\hat{\boldsymbol{x}}, \hat{\boldsymbol{y}}),
$$

where $E_{L}$ is the sampling efficiency of the sampling scheme and $\Delta(\hat{\boldsymbol{x}}, \hat{\boldsymbol{y}})$ is given in (9). We note that the mesh norm is also referred to as the covering radius as the spherical caps of radius equal to mesh norm and centered at sampling points cover the whole sphere. It is desirable to design a sampling scheme that minimizes the mesh norm [10]. For points of an extremal system for a band-limit $L$, Reimer [12] obtained an upper bound on the mesh norm of any system of points associated with positive weight cubature rule for a band-limit $L$. The upper bound is $\lambda(\mathfrak{S}) \leq 2 j_{o} /(L-1) \approx 4.8097 /(L-1)$, where $j_{o}$ is the smallest positive zero of the Bessel function $J_{o}$. It is worth noting here that we have incorporated the sampling efficiency in the formulation of mesh norm in (11). This is due to the fact that the sampling schemes under consideration require different number of samples for the representation of band-limited signal and therefore have different sampling efficiency.

Analysis: For each of the sampling schemes, we numerically compute the mesh norm by 1 ) randomly taking the $M$ number of uniformly distributed number of points on the sphere, 2) taking the minimum distance between the sampling

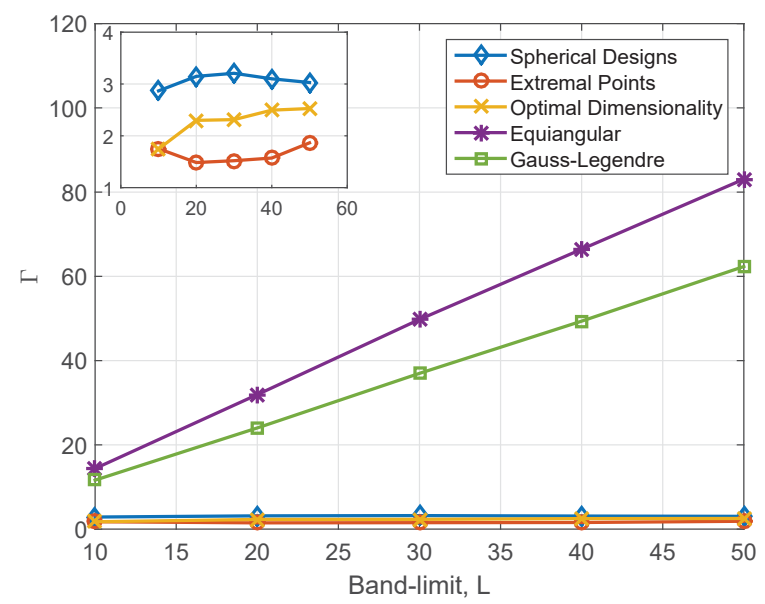

Fig. 4: Mesh ratio $\Gamma(\mathfrak{S})$, given in (12), for band-limits $10 \leq$ $L \leq 50$ and different sampling schemes.

points and the randomly chosen points on the sphere and 3) then obtaining the maximum over the minimum distances. We choose $M$ such that the numerically computed mesh norms by taking $M$ random points and $2 M$ random points do not differ more than $1 \%$. We plot the mesh norm for different sampling schemes in Fig. 3. It is evident that extremal system of points has the smallest mesh norm. As compared to equiangular and GL sampling schemes, spherical designs and optimal dimensionality sampling schemes have smaller mesh norm.

\section{Mesh Ratio}

Mesh ratio is the ratio of the covering to the packing radius of the identical spherical caps on the surface of a sphere. For a set $\mathfrak{S}$ of sampling points on the sphere, we define the mesh ratio as

$$
\Gamma(\mathfrak{S})=\frac{2 \lambda(\mathfrak{S})}{\sigma_{n}(\mathfrak{S})}>1,
$$

where we have used normalized geodesic distance in the formulation of mesh ratio as we also normalize the mesh norm with the sampling efficiency. Since the mesh ratio serves as a good measure of the quality of the uniform distribution of points, the sampling schemes should have smaller mesh norm.

Analysis: In Fig. 4, we plot the mesh ratio for different sampling schemes and different band-limits, where we observe that the mesh ratio for equiangular and GL sampling schemes increase with the band-limit, whereas the mesh ratio remains (almost) constant with the increase in band-limit. It can also be observed that the extremal system sampling scheme has the smallest mesh ratio.

\section{E. Riesz s-energy}

The Riesz $s$-energy $(s>0)$ of a set $\mathfrak{S}$ of points of the sampling scheme on the unit sphere may be defined as [23][25]

$$
\tilde{E}_{s}=\sum_{\hat{\boldsymbol{x}}, \hat{\boldsymbol{y}} \in \mathfrak{S} \hat{\boldsymbol{x} \neq \hat{\boldsymbol{y}}}} \frac{E_{L}}{2(\Delta(\hat{\boldsymbol{x}}, \hat{\boldsymbol{y}}))^{s}},
$$


where $\Delta(\hat{\boldsymbol{x}}, \hat{\boldsymbol{y}})$ is given in (9), $E_{L}$ is the sampling efficiency of the sampling scheme and we have normalized the Riesz energy measure by a factor of two as the distance for each distinct pair of sampling points appears twice in the summation. We also normalize with the sampling efficiency for a meaningful comparison. We note that the $s$-energy serves as a measure of the uniform distribution of points. Although this is not the focus of this work, we note that the sampling schemes have been proposed in the literature to determine sampling points that minimize their $s$-energy [24]-[26]. Here, we are only interested in analysing the $s$-energy for the sampling schemes that permit accurate computation of SHT.

Analysis: We compare the Riesz $s$-energy measure for different sampling schemes and different band-limits in Fig. 5 for $s=1$ and Fig. 5 for $s=2$. For $s=2, s$-energy measure is referred to as the potential energy [23]. It is evident that the extremal system, spherical design and optimal dimensionality sampling schemes have almost equal values of Riesz $s$-energy for $s=1$ and $s=2$. The uniform geometrical distribution of both these schemes is presented in Fig. 2, Fig. 3 and Fig. 4. Equiangular and GL sampling schemes have same trend for energy as spherical designs for $s=1$. But, as $s$ is increased, $s$-energy for both equiangular and GL sampling points goes away from the extremal system sampling scheme.

\section{F. Discussion}

Among the geometrical properties analysed for different sampling schemes, sampling efficiency, mesh norm and Riesz $s$-energy encapsulates the other properties and therefore serve as the measures of the uniform distribution of sampling points. Analysis of geometrical properties of the sampling scheme reveals that the mesh ratio and $s$-energy grow with the band-limit for the equiangular and Gauss-Legendre sampling schemes which is a consequence of the fact that these sampling schemes require dense sampling at the poles. In contrast, the optimal dimensionality, spherical design and extremal systems sampling schemes exhibit desired geometrical properties. Furthermore, the optimal dimensionality and extremal system sampling schemes have almost twice of the sampling efficiency attained by equiangular, Gauss-Legendre and spherical design sampling schemes. The mesh ratio achieved by optimal dimensionality is a little higher than extremal system, yet, it is very small compared to the equiangular schemes.

Sampling points of extremal systems have not been determined for large band-limits as the problem of finding these points becomes ill-conditioned and computationally expensive for large band-limits [10]. As mentioned earlier, extremal systems sampling points have only been computed for bandlimits up to $L=192$. As the problem becomes ill-conditioned for large band-limits, the accuracy of the computation of SHT degrades with the increase in the band-limit. To compare the accuracy of computation of SHT of a band-limited signal from its samples taken over the extremal system and optimaldimensionality sampling schemes, we conduct a numerical experiment and compute the maximum absolute error between the original spherical harmonic coefficients and reconstructed

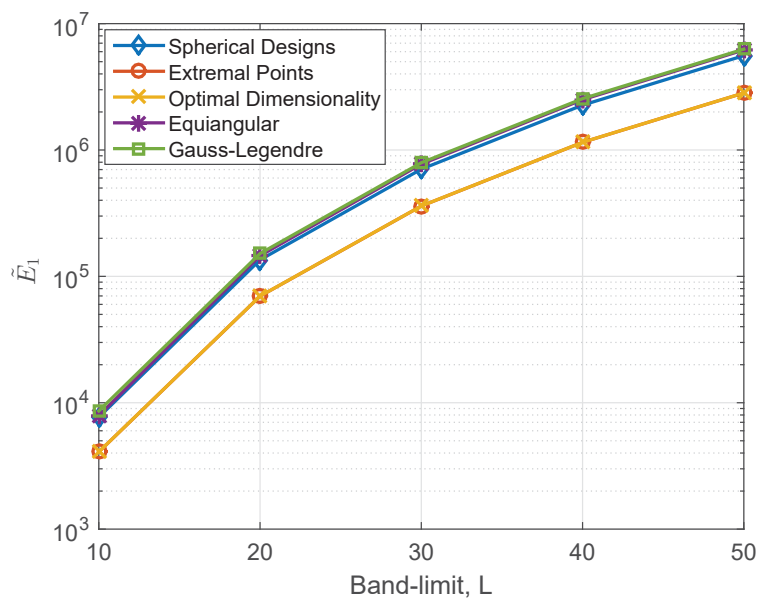

Fig. 5: Riesz energy $\tilde{E}_{s}$, given in (13), for $s=1$, band-limits $10 \leq L \leq 50$ and different sampling schemes.

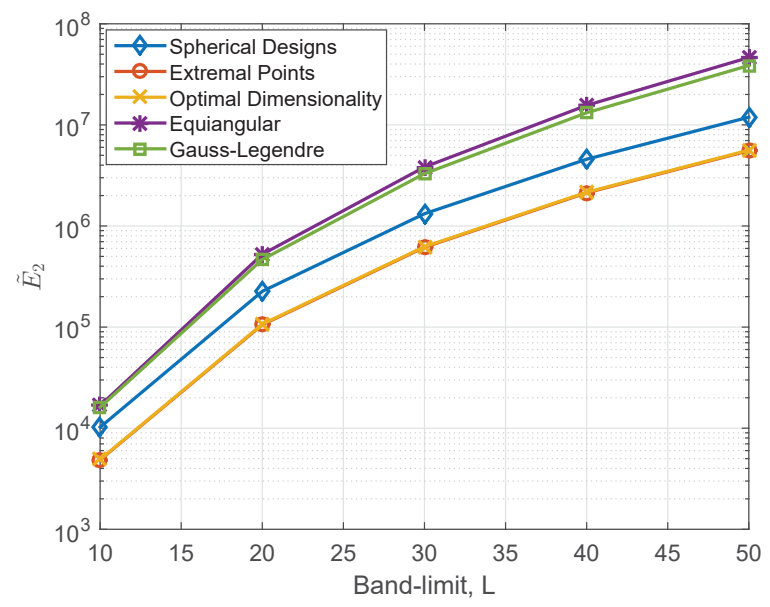

Fig. 6: Riesz energy $\tilde{E}_{s}$, given in (13), for $s=2$, band-limits $10 \leq L \leq 50$ and different sampling schemes.

spherical harmonic coefficients. In our experiment, 1) we randomly generate the spherical harmonic coefficients $(g)_{\ell}^{m}$ of the band-limited signal $g \in \mathcal{H}_{L}$ with real and imaginary parts uniformly distributed in $(-1,1), 2)$ we obtain the signal in the spatial domain over the samples of the respective sampling scheme using spherical harmonic expansion given in (2), 3) we compute the SHT using the samples of the signal [9], [10]. We carry out this experiment for $L=16,32$ and 64 and obtain the maximum error on the order of $10^{-15}, 10^{-14}$ and $10^{-14}$, and, $10^{-16}, 10^{-15}$ and $10^{-15}$ for extremal points sampling scheme and optimal-dimensionality sampling scheme respectively, illustrating that the optimaldimensionality sampling scheme enables accurate computation of SHT for the representation of band-limited signal for large band-limits. Furthermore, the SHT associated with the extremal system sampling scheme is based on least-squares approach, and therefore the complexity to compute SHT of the band-limited signal $g \in \mathcal{H}_{L}$ is $O\left(L^{6}\right)$. In comparison, 
the SHT for optimal dimensionality sampling scheme has the complexity $O\left(L^{3.37}\right)$. Summarizing our analysis, we propose that the extremal system sampling scheme is suitable for applications [27], [28] where the signals have smaller bandlimits $(L=10-50)$ due to superior geometrical properties. However, optimal dimensionality sampling scheme is better suited for applications [29], [30] where the data-sets or signals are supported by large band-limits $(L=1000-2000)$.

\section{Conclusions}

We have carried the comparative analysis of the geometrical properties of those sampling schemes that support the accurate representation of band-limited signals on the sphere. These schemes included equiangular sampling, Gauss-Legendre (GL) quadrature based sampling, optimal-dimensionality sampling, sampling points of extremal systems and spherical design. We have focused on analysing sampling efficiency, minimum geodesic distance, mesh norm, mesh ratio and Riesz $s$-energy for these sampling schemes. As all the schemes required different number of samples for the representation of the bandlimited signal on the sphere, we have introduced sampling efficiency in the formulation of mesh norm and Riesz $s$ energy in order to carry out a meaningful comparison. We have illustrated that the optimal dimensionality, extremal system and spherical design sampling schemes have a uniform distributions and the points are well separated on the sphere. Equiangular and GL sampling schemes exhibit poor geometrical properties due to the dense sampling near the poles. Extremal system sampling scheme has superior geometrical properties, which we propose to use for the representation of band-limited signal at small band-limits. However, the accuracy of computation of SHT degrades and computational complexity to compute SHT increases with the band-limit, due to which we propose to use optimal dimensionality sampling scheme for large band-limits as it allows exact computation of SHT.

\section{REFERENCES}

[1] A. Amirbekyan, V. Michel, and F. J. Simons, "Parametrizing surface wave tomographic models with harmonic spherical splines," Geophys. J. Int., vol. 174, pp. 617-628, Aug. 2008.

[2] R. Ng, R. Ramamoorthi, and P. Hanrahan, "Triple product wavelet integrals for all-frequency relighting," ACM Trans. Graph., vol. 23, no. 3, pp. 477-487, Aug. 2004

[3] G. Lavaux and B. D. Wandelt, "Fast and optimal cosmic microwave background lensing using statistical interpolation on the sphere," Astrophys. J., Suppl. Ser., vol. 191, no. 1, p. 32, Oct. 2010.

[4] N. Jarosik, C. L. Bennett, J. Dunkley, B. Gold, M. R. Greason, M. Halpern, R. S. Hill, G. Hinshaw, A. Kogut, E. Komatsu, D. Larson, M. Limon, S. S. Meyer, M. R. Nolta, N. Odegard, L. Page, K. M. Smith, D. N. Spergel, G. S. Tucker, J. L. Weiland, E. Wollack, and E. L. Wright, "Seven-year wilkinson microwave anisotropy probe (wmap) observations: Sky maps, systematic errors, and basic results," Astrophys. J., Suppl. Ser., vol. 192, no. 2, p. 14, Jan. 2011.

[5] M. K. Chung, K. M. Dalton, L. Shen, A. C. Evans, and R. J. Davidson, "Weighted fourier series representation and its application to quantifying the amount of gray matter," IEEE Trans. Med. Imag., vol. 26, no. 4, pp. 566-581, Apr. 2007.

[6] W. Zhang, M. Zhang, R. A. Kennedy, and T. D. Abhayapala, "On high-resolution head-related transfer function measurements: An efficient sampling scheme," IEEE/ACM Trans. Audio, Speech, Language Process., vol. 20, no. 2, pp. 575-584, Feb. 2012.
[7] T. S. Pollock, T. D. Abhayapala, and R. A. Kennedy, "Introducing space into mimo capacity calculations," Telecom. Sys., vol. 24, no. 2, pp. 415436, Oct. 2003.

[8] R. A. Kennedy and P. Sadeghi, Hilbert Space Methods in Signal Processing. Cambridge, UK: Cambridge University Press, Mar. 2013.

[9] Z. Khalid, R. A. Kennedy, and J. D. McEwen, "An optimaldimensionality sampling scheme on the sphere with fast spherical harmonic transforms," IEEE Trans. Signal Process., vol. 62, no. 17, pp. 4597-4610, Sep. 2014

[10] I. H. Sloan and R. S. Womersley, "Extremal systems of points and numerical integration on the sphere," Adv. Comput. Math., vol. 21, no. 1, pp. 107-125, Jul. 2004.

[11] I. H. Sloan and R. S. Womersley, "A variational characterisation of spherical designs," J. Approx. Theory, vol. 159, no. 2, pp. 308 - 318, Aug. 2009.

[12] M. Reimer, "Spherical polynomial approximations: a survey," MATH RES, vol. 107, pp. 231-252, Nov. 1999.

[13] J. Driscoll and D. Healy, "Computing fourier transforms and convolutions on the 2-sphere," Adv. Appl. Math., vol. 15, no. 2, pp. $202-250$, Jun. 1994.

[14] R. G. Crittenden and N. G. Turok, "Exactly azimuthal pixelizations of the sky," Arxiv preprint astro-ph/9806374, Jun. 1998.

[15] J. D. McEwen and Y. Wiaux, "A novel sampling theorem on the sphere," IEEE Trans. Signal Process., vol. 59, no. 12, pp. 5876-5887, Dec. 2011

[16] K. M. Huffenberger and B. D. Wandelt, "Fast and exact spin-s spherical harmonic transforms," Astrophys. J., Suppl. Ser., vol. 189, no. 2, p. 255, Aug. 2010.

[17] M. J. Mohlenkamp, "A fast transform for spherical harmonics," J. Fourier Anal. Appl., vol. 5, no. 2, pp. 159-184, Mar. 1999.

[18] J. A. R. Blais and M. A. Soofi, Spherical Harmonic Transforms Using Quadratures and Least Squares. Berlin, Heidelberg: Springer Berlin Heidelberg, May. 2006, pp. 48-55.

[19] M. Reimer, Constructive theory of multivariate functions: with an application to tomography. BI-Wissenschaftverlag, 1990.

[20] X. Chen, A. Frommer, and B. Lang, "Computational existence proofs for spherical $t$-designs," Numer. Math., vol. 117, no. 2, pp. 289-305, Feb. 2011.

[21] A. G. Doroshkevich, P. D. Naselsky, O. V. Verkhodanov, D. I. Novikov, V. I. Turchaninov, I. D. Novikov, P. R. Christensen, and Chiang, "Gauss Legendre Sky Pixelization (GLESP) for CMB maps," Int. J. Mod. Phys. D., vol. 14, no. 02, pp. 275-290, Feb. 2005.

[22] D. S. Seljebotn and H. K. Eriksen, "Sympix: A spherical grid for efficient sampling of rotationally invariant operators," Astrophys. J., Suppl. Ser. vol. 222, no. 2, p. 17, Apr. 2015.

[23] P. Leopardi, "Distributing points on the sphere: partitions, separation, quadrature and energy," Ph.D. dissertation, University of New South Wales, Apr. 2007

[24] J. S. Brauchart, "About the second term of the asymptotics for optimal riesz energy on the sphere in the potential-theoretical case," INTEGR. TRANSF. SPEC. F., vol. 17, no. 5, pp. 321-328, Jan. 2006.

[25] A. Kuijlaars, E. Saff, and X. Sun, "On separation of minimal riesz energy points on spheres in euclidean spaces," J. Comput. Appl. Math., vol. 199, no. 1, pp. 172-180, Feb. 2007.

[26] D. Hardin and E. Saff, "Minimal riesz energy point configurations for rectifiable d-dimensional manifolds," Adv. Math., vol. 193, no. 1, pp. 174-204, May. 2005.

[27] A. P. Bates, Z. Khalid, and R. A. Kennedy, "An optimal dimensionality sampling scheme on the sphere with accurate and efficient spherical harmonic transform for diffusion mri," IEEE Signal Process. Lett., vol. 23, no. 1, pp. 15-19, Jan. 2016.

[28] A. P. Bates , Z. Khalid, and R. A. Kennedy, "Novel sampling scheme on the sphere for head-related transfer function measurements," IEEE/ACM Trans. Audio, Speech, Language Process., vol. 23, no. 6, pp. 1068-1081, Jun. 2015.

[29] N. Sneeuw, "Global spherical harmonic analysis by least-squares and numerical quadrature methods in historical perspective," Geophys. J. Int., vol. 118, no. 3, pp. 707-716, Sep. 1994.

[30] Y. Wiaux, L. Jacques, and P. Vandergheynst, "Fast spin 2 spherical harmonics transforms and application in cosmology," J. Comput, Phys., vol. 226, no. 2, pp. 2359-2371, Oct. 2005. 